\title{
Cosmic-Ray Isotopic Composition Results from the ACE Mission
}

\author{
M. E. Wiedenbeck \\ Jet Propulsion Laboratory, California Institute of Technology \\ MS 169-327, 4800 Oak Grove Dr., Pasadena, CA 91109
}

\begin{abstract}
The Cosmic Ray Isotope Spectrometer (CRIS) instrument on the Advanced Composition Explorer (ACE) mission has been making high precision measurements of the isotopic and elemental composition of galactic cosmic rays over the past two years. These observations have been used to investigate a range of topics including: 1) the nucleosynthetic processes that produced the cosmic-ray source material, 2) the processes responsible for fractionation of the elements in this material, 3) the time that elapsed between nucleosynthesis and acceleration to high energies, and 4) the confinement time of cosmic rays in the Galaxy. Highlights from CRIS investigations reported to date are summarized.
\end{abstract}

\section{INTRODUCTION}

The Advanced Composition Explorer (ACE) spacecraft was launched on $25 \mathrm{Au}$ gust 1997 into a halo orbit about the L1 Lagrange point, $1.5 \times 10^{6} \mathrm{~km}$ sunward of the Earth [1]. Among the instruments it carries is the Cosmic Ray Isotope Spectrometer (CRIS) [2] which covers the energy range from $\sim 50$ to $\sim 500 \mathrm{MeV} /$ nucleon and samples predominantly galactic cosmic-ray material. CRIS identifies the charge and mass of detected nuclei using the $d E / d x$ versus total energy technique. Energy loss measurements are made in 4 stacks of large-area silicon solid-state detectors, and particle trajectories are derived from measurements made in a scintillating optical fiber hodoscope in front of the silicon stacks. An important feature of this instrument is its large geometrical acceptance, $\sim 250 \mathrm{~cm}^{2} \mathrm{sr}$, which is a factor $\sim 20$ larger than has been available with previous space instruments of this kind.

\section{SOURCE COMPOSITION OF Fe, Co, AND Ni}

The study of the nucleosynthesis of cosmic-ray source material is best addressed using nuclides that require minimal corrections for contributions from secondaries produced by nuclear fragmentation during transport through the Galaxy. A number of such species are found in the upper portion of the iron-cobalt-nickel group,

CP516, $26^{\text {th }}$ International Cosmic Ray Conference, edited by B. L. Dingus, et al. (1) 2000 American Institute of Physics 1-56396-939-4/00/\$17.00 
$57 \leq M \leq 64$, where secondaries cannot be produced by fragmentation of the dominant isotope ${ }^{56} \mathrm{Fe}$. Furthermore, iron-group nuclides are of particular interest because their abundances are sensitive to the final stages of hydrostatic burning in stars and to the alterations that occur in the supernova explosions that follow.
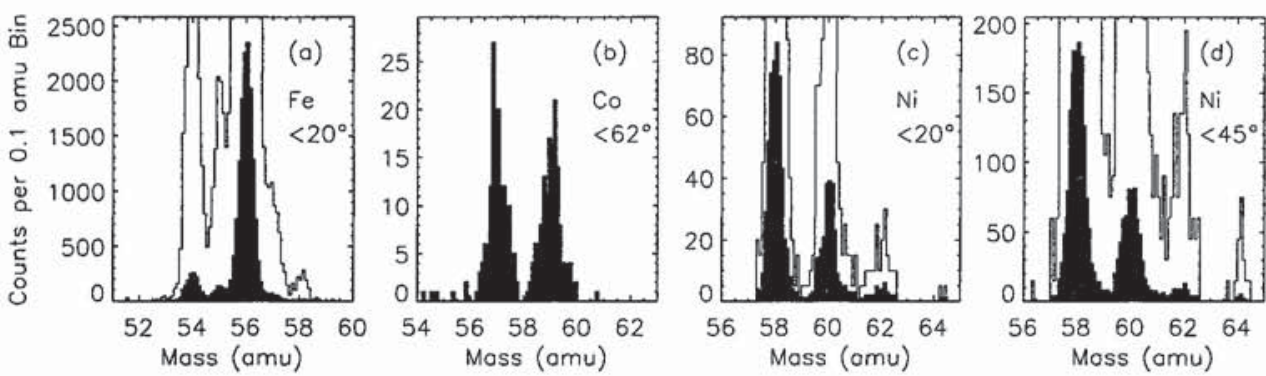

FIGURE 1. CRIS mass histograms. Unfilled histograms have vertical scales expanded to better show the rare isotopes. Data selection included cuts on angle of incidence, $\theta$, to optimize the trade off between statistics and resolution (which ranges from $\sim 0.23 \mathrm{amu}$ at small angles to approximately twice this value at $\left.\theta=45^{\circ}\right)$.

Figure 1 shows mass histograms obtained from CRIS for the elements iron, cobalt, and nickel. Particles incident at small angles $\left(\theta<20^{\circ}\right)$ from the detector normal were selected to obtain a high resolution data set from which the abundances of ${ }^{59} \mathrm{Ni},{ }^{61} \mathrm{Ni}$, and ${ }^{62} \mathrm{Ni}$, could be derived. A wider angle cut were used to retain adequate statistics for ${ }^{64} \mathrm{Ni}$ which has a very low abundance but is separated by $2 \mathrm{amu}$ from the lighter isotopes.

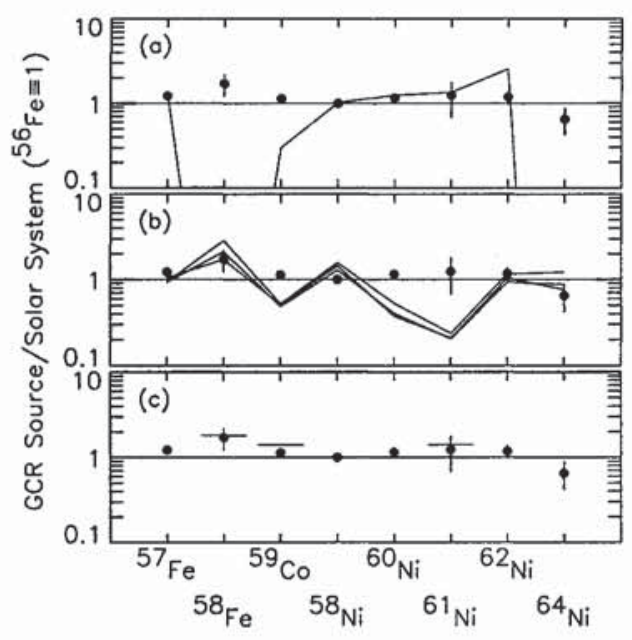

FIGURE 2. Comparison of derived GCR source abundances with model predictions: (a) SN II, (b) SN Ia, (c) Wolf-Rayet+ISM.
Figure 2 shows source abundances derived from the CRIS data as the points plotted in each panel. The abundances are shown relative to the corresponding abundances in solar system material [3]. The most notable feature seen in this comparison is the great similarity between the cosmic-ray source and solar system compositions: differences do not exceed a few tens of percent, in spite of the fact that absolute abundances range over more than a factor of 1000 . The data in Figure 2 do suggest the possibility of statistically-significant differences from solar system composition, particularly for ${ }^{58} \mathrm{Fe}$, but these should not be taken too seriously 
until possible systematic uncertainties have been thoroughly investigated.

The lines in the three panels show the abundance patterns expected from several possible sources of cosmic rays. The line in panel (a) shows calculated yields from Type II supernovae (hereafter, SN II) which result from the core collapse of massive stars $[4,5]$. These abundances include contributions from a range of masses of the pre-supernova stars, with the yields weighted by a Salpeter initial mass function. A striking feature of these calculations is the negligible abundances predicted for the most neutron-rich nuclides, ${ }^{58} \mathrm{Fe}$ and ${ }^{64} \mathrm{Ni}[6]$.

The lines in panel (b) show yields calculated from several models of Type Ia supernovae (hereafter, SN Ia), which result from deflagration/detonation of white dwarf stars which have accreted a critical mass of material from a binary companion [4]. These SN Ia yields include near-solar abundances of ${ }^{58} \mathrm{Fe}$ and ${ }^{64} \mathrm{Ni}$, but are somewhat deficient in ${ }^{59} \mathrm{Co},{ }^{60} \mathrm{Ni}$, and ${ }^{61} \mathrm{Ni}$. Taken together, the comparisons with SN II and SN Ia yields suggest that the cosmic-ray source most likely contains contributions from both types of supernovae, and the similarity to solar system composition suggests that the relative contributions to that mix must be close to those found in solar system material where SN Ia are thought to contribute $~ 15 \%$ of the total material but more than half of the material in the iron group [5].

If correct, the calculations indicating that ${ }^{58} \mathrm{Fe}$ and ${ }^{64} \mathrm{Ni}$ are not significantly produced in SN II [6] would lead to the conclusion that cosmic-ray source material contains appreciable contributions from sources other than SN II. However, another set of calculations [7] indicate that these very neutron-rich nuclides can be produced in near-solar proportions in SN II. The resolution of these differences between the theoretical calculations is very important for the interpretation of the cosmic-ray composition data. Recently reported comparisons of the two sets of calculations [8] attempt to identify and resolve major discrepancies, but the question of whether SN II can be the major source of ${ }^{58} \mathrm{Fe}$ and ${ }^{64} \mathrm{Ni}$ remains to be answered.

At present the only well-established isotopic difference between the composition of the cosmic-ray source and the solar system is the large excess of ${ }^{22} \mathrm{Ne}$ in the former sample (e.g., $[9,10]$ and references therein). It has been proposed [11] that this anomaly results from a small (few percent) contribution from Wolf-Rayet star winds, which contain large excesses of this isotope, to the general mix of interstellar matter that gets accelerated to cosmic-ray energies. Such a contribution should contain smaller excesses of a variety of neutron-rich nuclides since neutrons produced from the ${ }^{22} \mathrm{Ne}(\alpha, \mathrm{n})^{25} \mathrm{Mg}$ reaction would lead to s-processing (slow neutron addition) of material in the Wolf-Rayet star. The horizontal line segments in panel (c) show the iron-group cosmic-ray source composition that could be expected from such a model [12]. (Excesses of nuclides for which no line is shown are predicted be less than $20 \%$.) The consistency between the predicted excess of ${ }^{58} \mathrm{Fe}$ and the value derived from the CRIS observations is suggestive, but the CRIS measurement of ${ }^{59} \mathrm{Co}$ does not show the predicted enhancement. A more thorough treatment of the systematic uncertainties associated with the derived source abundances is needed to help decide whether a Wolf-Rayet contribution is clearly supported. 


\section{COMPOSITION OF TRANS-NICKEL SPECIES}

Although abundances of the elements heavier than nickel decrease rapidly with increasing atomic number, CRIS collects adequate statistical samples for exploratory studies of the elemental and isotopic composition of elements with $29 \leq Z \lesssim 34$ [13]. Figure 3 shows the histogram of calculated charges for the nuclei collected in this charge range. In the $d E / d x$ versus total energy technique the calculated quantity used for particle identification is a function of both charge and, more

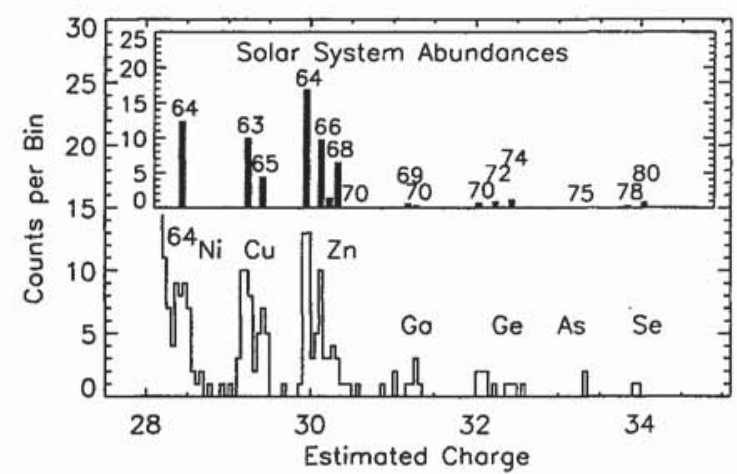

FIGURE 3. CRIS measurements of nuclides beyond nickel [13]. wẹakly, mass [2]. Thus the peak shapes for the elements exhibit structure indicative of the isotopes that are present, as can be seen in the cases of ${ }_{29} \mathrm{Cu}$ and ${ }_{30} \mathrm{Zn}$. The inset panel is a bar chart showing the abundances found in solar system material [3] plotted to facilitate comparison with the CRIS measurements. The isotopic composition of $\mathrm{Cu}$ and $\mathrm{Zn}$ continues the general pattern of solar-like abundances found for $\mathrm{Fe}$ and $\mathrm{Ni}$.

The elemental abundances of $\mathrm{Cu}$ and $\mathrm{Zn}$ are approximately equal in the cosmicray sample, whereas in the solar system the $\mathrm{Zn}$ abundance is $2.4 \times$ that of $\mathrm{Cu}$. This simply reflects the well-established elemental fractionation which is correlated with the first ionization potential (FIP) and also with the volatility of the elements $[14,15]$, and is consistent with previous cosmic-ray observations at higher energies [16]. An important unresolved question for understanding cosmic-ray origins is whether the elemental fractionation is actually associated with FIP or with volatility, since the fractionation mechanism should be indicative of the site and physical processes associated with cosmic-ray production. A small number of elements have potential for distinguishing which is the parameter controlling the fractionation by virtue of having significantly greater volatility than is typical for a given FIP value [15]. For example, the FIP value of Ge is nearly identical to that of Fe but these elements have condensation temperatures $\sim 800 \mathrm{~K}$ and $\sim 1300 \mathrm{~K}$, respectively. Other possible discriminators between FIP- and volatility-controlled fractionation include $\mathrm{Cu}, \mathrm{Zn}$, and $\mathrm{Ga}$.

Figure 4 shows the source abundances derived from the CRIS data as a function of FIP for elements with $26 \leq Z \leq 34$, normalized to solar system abundances [3], together with previously reported results from the HEAO-C2 experiment [17] for elements with $8 \leq Z \leq 20$. With present uncertainties, which are predominantly statistical for $Z>28$, the CRIS data appear reasonably consistent with a correlation controlled by FIP. In particular, comparing elements having similar FIP values 
there do not appear to be significant depletions of $\mathrm{Ge}$ relative to $\mathrm{Fe}, \mathrm{Cu}$ relative to $\mathrm{Mg}$, or $\mathrm{Ga}$ relative to $\mathrm{Al}$, as one would expect from a volatility-related fractionation process. The interpretation of the $\mathrm{Zn}$ abundance is complicated by the fact that its FIP value lies in the transition between high and low-FIP species. Any conclu-

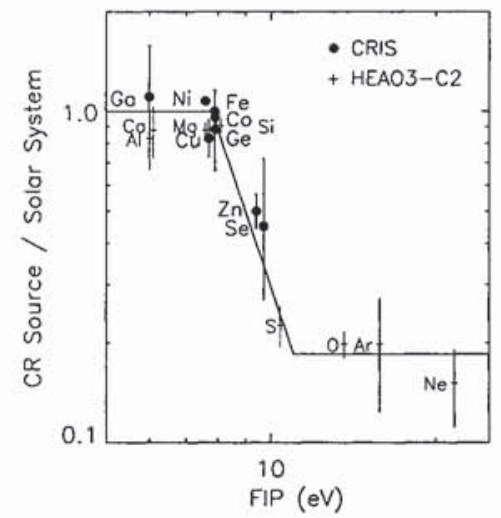

FIGURE 4. Elemental fractionation of the GCR source relative to solar system composition [13]. sion about the enhancement or depletion of $\mathrm{Zn}$ relative to the general trend of other elemental abundances versus FIP depends on the shape and location of the transition, which remain somewhat uncertain.

At higher energies, combined results from Ariel 6 and HEAO-C3 [18] indicated a depletion of $\mathrm{Ge}$ relative to $\mathrm{Fe}$ by a factor $\sim 1.65 \pm 0.15$. The CRIS result is marginally inconsistent with this value, but until the CRIS statistical errors can be reduced it appears premature to conclude either that there is definitely a difference between these measurements or that the CRIS observations favor a fractionation mechanism related to FIP rather than volatility.

\section{TIME DELAY BEFORE ACCELERATION}

It has been pointed out $[19,20]$ that information about the time that elapses between nucleosynthesis of the cosmic-ray source material and its acceleration to high energies can be obtained from the abundances of primary radionuclides that are produced in supernova explosions and can decay only by orbital electron capture. At cosmic-ray energies above a few hundred $\mathrm{MeV} /$ nucleon iron-group ions will be completely stripped of their electrons as they pass through the interstellar medium. Lacking any electrons to capture, these nuclei become stable. However, if the nuclei reside in the thermal ejecta of the supernova for a time long compared to their halflife, electron capture decays will have time to occur, transforming the radioactive nuclei into their daughter products.

The most useful nuclides for such a study are ${ }^{59} \mathrm{Ni}\left(7.6 \times 10^{5} \mathrm{yr}\right.$ halflife $)$ and ${ }^{57} \mathrm{Co}$ (0.74 yr halflife) since these species are not abundantly produced as secondary cosmic rays. As seen in Figure $1,{ }^{59} \mathrm{Ni}$ is nearly absent in the CRIS data while its daughter, ${ }^{59} \mathrm{Co}$, is clearly present. Figure 5 shows a quantitative comparison between the measured abundances of these two isotopes (hatched regions) and values cal : lated for various assumed acceleration delay times (abscissa) and fractions of the primary mass- 59 material synthesized as ${ }^{59} \mathrm{Ni}$ (labels on the curves). The dashed lines indicate the secondary contributions produced during cosmicray transport. The abundances of both ${ }^{59} \mathrm{Ni}$ and ${ }^{59} \mathrm{Co}$ are consistent with the complete decay of the primary ${ }^{59} \mathrm{Ni}$ prior to acceleration. Models of the nucleosyn- 
thesis and ejection of heavy nuclei in Type II supernova explosions [7] indicate that the ejected mass-59 material should include at least $\sim 25 \%{ }^{59} \mathrm{Ni}$, and probably significantly more. Ejecta from Type Ia supernovae should also contain signif-
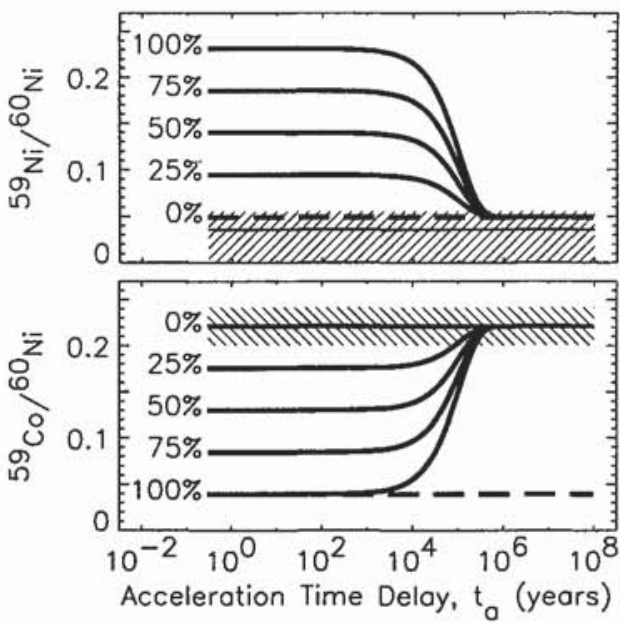

FIGURE 5. Evolution of ${ }^{59} \mathrm{Ni}$ and ${ }^{59} \mathrm{Co}$ abundances between nucleosynthesis and cosmic-ray acceleration (see text for details). icant fractions of ${ }^{59} \mathrm{Ni}[21]$. Thus one concludes that the absence of primary ${ }^{59} \mathrm{Ni}$ in the arriving cosmic rays is due to a delay time $\gtrsim 10^{5} \mathrm{yr}$ between nucleosynthesis and acceleration. Further details of the ACE measurements of ${ }^{59} \mathrm{Ni}$ and ${ }^{59} \mathrm{Co}$ and the analysis of the acceleration time delay are discussed in a recent publication [22].

Analysis of CRIS measurements of the electron-capture nuclide ${ }^{57} \mathrm{Co}$ and its daughter, ${ }^{57} \mathrm{Fe}$, shows that the abundances of these isotopes are also consistent with the complete decay of the electron-capture primary. This is expected on the basis of the ${ }^{59} \mathrm{Ni}$ result since the halflife of ${ }^{57} \mathrm{Co}$ is much shorter than that of ${ }^{59} \mathrm{Ni}$.

\section{SECONDARY BETA-DECAY CLOCKS}

The confinement time of cosmic rays in the Galaxy can be derived from the "surviving fraction" (ratio of the observed abundance to the abundance expected in the absence of decay) of a secondary nuclide that decays by $\beta^{+}$or $\beta^{-}$emission. Until recently the only precise confinement time determinations were for ${ }^{10} \mathrm{Be}$ (e.g., [23] and references therein), but as a result of high resolution measurements of heavy isotopes on Ulysses [24-27] and ACE [28], accurate values are now available for ${ }^{26} \mathrm{Al},{ }^{36} \mathrm{Cl}$, and ${ }^{54} \mathrm{Mn}$ as well. Taken together, the CRIS data for ${ }^{10} \mathrm{Be},{ }^{26} \mathrm{Al}$, and ${ }^{36} \mathrm{Cl}$ indicate a mean cosmic-ray confinement time $\sim 17 \pm 4 \mathrm{Myr}$ in a leaky-box propagation model. A larger value, $\sim 27 \mathrm{Myr}$, is obtained for ${ }^{54} \mathrm{Mn}$, but with a large uncertainty due to the poorly known $\beta^{-}$halflife of this isotope.

For nuclides propagating in a homogeneous medium with halflife, $T_{1 / 2}$, short compared to the confinement time, the surviving fraction will increase proportional to $T_{1 / 2}$, reflecting the increasing probability that the nuclei can reach the neighborhood of the solar system before decaying. If, however, the solar system resides in a "bubble" (typical dimension $L$ ) in which the density of interstellar gas is significantly lower than that of the average interstellar medium, the production of secondary cosmic rays will be diminished inside this bubble. In this case the 
surviving fractions will be reduced for those nuclides with halflives comparable to or less than the time required to diffuse across the bubble, $T_{1 / 2} \lesssim L^{2} / D$ where $D$ is the particles' diffusion coefficient.
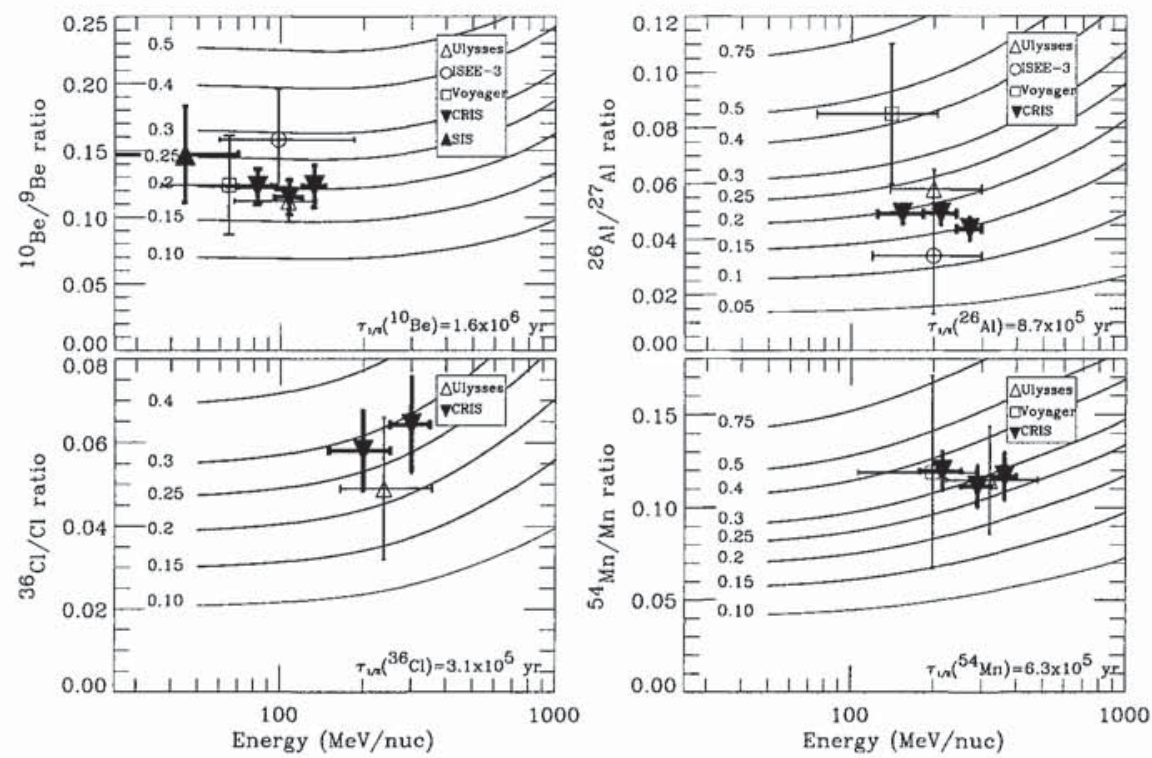

FIGURE 6. Comparison of secondary clock isotope measurements with energy dependences calculated using a leaky-box model and $555 \mathrm{MV}$ solar modulation.

Data from ACE $[28,29]$ and Ulysses [24-27] indicate that the surviving fractions of ${ }^{26} \mathrm{Al},{ }^{36} \mathrm{Cl},{ }^{54} \mathrm{Mn}$ are generally consistent with the values predicted on the basis of the ${ }^{10} \mathrm{Be}$ surviving fraction and a leaky-box propagation model. However, the total range of halflives among these four nuclides is only a factor $\sim 5$ and large deviations are not expected for most realistic propagation models that have been proposed [23]. A much more stringent test could be obtained from measurements of a finite surviving fraction of ${ }^{14} \mathrm{C}$, which has a halflife of only $5730 \mathrm{yr}$, a factor of 280 less than that of ${ }^{10} \mathrm{Be}$. A small amount of ${ }^{14} \mathrm{C}$ is seen in the CRIS data, but until contributions from local production of ${ }^{14} \mathrm{C}$ in the instrument can be fully assessed, one can only quote an upper limit of $\sim 2 \%$ for the surviving fraction. This limit is not very stringent, but can be used to rule out extreme models in which cosmic rays traverse more than a few percent of their interstellar grammage in the immediate vicinity of the solar system.

Another test of propagation models can be obtained from the energy dependences of the surviving fractions of the various secondary radionuclides. One expects surviving fractions to increase going toward higher energies both because of relativistic time dilation of the halflife and because the higher velocity particles require less 
time to traverse a given distance. However, the quantitative predictions of the energy dependence of this increase differ between models [30], so measurements of this dependence should prove useful for distinguishing among the various possibilities. By subdividing the CRIS data it is possible to obtain precise measurements of the secondary clock isotopes at multiple energies which can then be compared with model predictions of the energy dependence. Figure 6 shows a comparison between the CRIS observations and leaky-box model calculations. The CRIS data (filled triangles pointing down) show no increase with energy, to within the measurement uncertainties. For ${ }^{26} \mathrm{Al}$ and ${ }^{54} \mathrm{Mn}$ the observed independence of energy appears marginally inconsistent with the leaky-box predictions and may indicate the need for a more physical propagation model. For example, models which take into account diffusion of cosmic rays in a thin galactic disk bounded by a thick, low-density halo tend to predict a weaker energy dependence of the surviving fractions [30]. Improved tests of such models should become possible as additional data collection reduces the CRIS statistical uncertainties and as new experiments [31] extend measurements of ${ }^{10} \mathrm{Be}$ to $\mathrm{GeV} /$ nucleon energies where differences between models should be much more pronounced.

\section{CONCLUSIONS}

The CRIS instrument on ACE is providing statistically-accurate, isotopicallyresolved measurements of essentially all nuclides up through the iron group in low-energy galactic cosmic rays. Building on previous work, these data support a picture in which cosmic rays are accelerated from old stellar or interstellar matter which was produced by essentially the same mix of nucleosynthesis processes as was solar system matter. The accelerated particles are confined in the Galaxy for $\sim 20 \mathrm{Myr}$ in a region with a mean density of a few tenths of an atom per $\mathrm{cm}^{3}$. At the present stage of analysis the CRIS data do not conclusively distinguish between FIP and volatility as the parameter controlling the elemental fractionation of cosmic rays.

CRIS data are being used for investigating a wide range of topics in addition to those discussed above. Examples include the energy dependence of the abundances of secondary electron capture nuclides as a probe of reacceleration or other energy changing processes; cosmic-ray elemental composition and spectra; short-term variations of the intensities of cosmic-ray nuclides; and, in conjunction with various deep-space missions, the radial dependence of cosmic-ray modulation. Much additional data analysis and modeling remains to be done to fully exploit the richness of the CRIS data set.

\section{ACKNOWLEDGMENTS}

The work summarized here was performed by the ACE/CRIS team: N. E. Yanasak, J. S. George, W. R. Binns, E. R. Christian, A. C. Cummings, P. L. 
Hink, R. A. Leske, M. Lijowski, J. Klarmann, S. M. Niebur, R. A. Mewaldt, E. C. Stone, and T. T. von Rosenvinge. This research was supported by NASA at the California Institute of Technology (under grant NAG5-6912), the Jet Propulsion Laboratory, the Goddard Space Flight Center, and Washington University.

\section{REFERENCES}

1. Stone, E. C., et al., Sp. Sci. Rev. 86, 1 (1998).

2. Stone, E. C., et al., Sp. Sci. Rev. 86, 283 (1998).

3. Anders, E., \& Grevesse, N. 1989, Geochim. Cosmochim. Acta, 53, 197 (1989).

4. Nomoto, K. et al., in Thermonuclear Supernovae, Ruiz-Lapuenta (ed.), Kluwer:Dordrecht, pp. 349-378 (1997).

5. Tsujimoto, T. et al., MNRAS 277, 945 (1995).

6. Thielemann, F.-K., Nomoto, K, \& Hashimoto, M., ApJ 460, 408 (1996).

7. Woosley, S. E., \& Weaver, T. A., Ap. J. Suppl. 101, 181 (1995).

8. Hoffman, R. D. et al., Ap. J. 521, 735 (1999).

9. DuVernois, M. A. et al., Ap. J. 466, 457 (1996).

10. Webber, W. R., Lukasiak, A., \& McDonald, F. B., Ap. J. 476, 766 (1997).

11. Cassé, M., \& Paul, J. A., Ap. J. 258, 860 (1982).

12. Prantzos, N. et al., Proc. 19th Int. Cosmic Ray Conf. (La Jolla) 3, 167 (1985).

13. George, J. S. et al., Proc. 26th Int. Cosmic Ray Conf. (Salt Lake City) 3, 13 (1999).

14. Meyer, J.-P., Ap. J. Suppl. 57, 173 (1985).

15. Meyer, J.-P., Drury, L., O'C, \& Ellison, D. C., Ap. J. 487, 182 (1997).

16. Byrnak, B. et al., Proc. 18th Int. Cosmic Ray Conf. (Bangalore) 2, 29 (1983).

17. Ferrando, P., J. Phys. G 19, S53 (1993).

18. Binns, W. R. et al., in Cosmic Abundances of Matter, C. J. Waddington (ed.), AIP: New York, 1989, p.147.

19. Cassé, M., \& Soutoul, A., Ap. J. (Letters) 200, L75 (1978).

20. Soutoul, A., Cassé, M., \& Juliusson, Ap. J. 219, 753 (1978).

21. Meyer, J.-P. \& Ellison, D. C., in LiBeB, Cosmic Rays, and Related X-and GammaRays, Ramaty et al. (eds.), Astro. Soc. Pacific Conf. Series, vol. 171, p. 187 (1999).

22. Wiedenbeck, M. E. et al., Ap. J. (Letters) 523, L61 (1999).

23. Ptuskin, V. S. \& Soutoul, A., Astron. Astroph. 337, 859 (1998).

24. DuVernois, M. A., Ap. J. 481, 247 (1997).

25. Connell, J. J., Ap. J. (Letters) 501, L59 (1998).

26. Connell, J. J., DuVernois, M. A., and Simpson, J. A., Ap. J. (Letters) 509, L97 (1998).

27. Simpson, J. A. \& Connell, J. J., Ap. J. (Letters) 497, L85 (1998).

28. Binns, W. R. et al., Proc. 26th Int. Cosmic Ray Conf. (Salt Lake City), 3, 21 (1999).

29. Yanasak, N. E. et al., Proc. 26th Int. Cosmic Ray Conf. (Salt Lake City), 3, 9 (1999).

30. Simon, M. \& Molnar, A., Proc. 26th Int. Cosmic Ray Conf. (Salt Lake City), 4, 211 (1999).

31. Mitchell, J. W., et al., Proc. 26th Int. Cosmic Ray Conf. (Salt Lake City), 3, 113 (1999). 